\title{
OSCILLATIONS OF THE SUNFLOWER EQUATION*
}

By

M. R. S. KULENOVIĆ (University of Sarajevo, Yugoslavia)

AND

G. LADAS (University of Rhode Island)

Abstract. Consider the delay differential equation

$$
\ddot{y}(t)+\alpha \dot{y}(t)+\beta f(y(t-r))=0,
$$

where $\alpha, \beta$, and $r$ are positive constants and $f$ is a continuous function such that

$$
u f(u)>0 \text { for } u \in[-A, B], u \neq 0 \text {, and } \lim _{u \rightarrow 0} \frac{f(u)}{u}=1 \text {, }
$$

where $A$ and $B$ are positive numbers. When $f(u)=\sin u,(*)$ is the so-called "sunflower" equation, which describes the motion of the tip of the sunflower plant.

We obtain necessary and sufficient conditions for the oscillation of all solutions of (*), whose graph lies eventually in the strip $R^{+} \times[-A, B]$, in terms of the characteristic equation of the linearized equation

$$
\ddot{z}(t)+\alpha \dot{z}(t)+\beta z(t-r)=0 .
$$

1. Introduction and preliminaries. In this paper we are concerned with the problem of oscillation of second-order delay differential equations (DDE) of the form

$$
\ddot{y}(t)+\alpha \dot{y}(t)+\beta f(y(t-r))=0, \quad t \geq t_{0},
$$

where $\alpha, \beta$, and $r$ are positive constants and $f$ is a real-valued continuous function such that

$$
u f(u)>0 \text { for } u \in[-A, B], u \neq 0,
$$

where $A$ and $B$ are given positive numbers and

$$
\lim _{u \rightarrow 0} \frac{f(u)}{u}=1 .
$$

An example of such an equation and a motivating force behind our results is the sunflower equation

$$
\ddot{y}(t)+\frac{a}{r} \dot{y}(t)+\frac{b}{r} \sin y(t-r)=0, \quad t \geq 0,
$$

*Received April 16, 1986. 
introduced in 1967 by Israelsson and Johnsson [1] as a model for the geotropic circumnutations of Helianthus annus and whose periodic solutions were studied by Somolinos [3] in 1978.

We are interested in solutions of Eq. (1) whose graph for $t$ sufficiently large lies in the strip $R^{+} \times[-A, B]$. For all such solutions we obtained necessary and sufficient conditions for their oscillations in terms of the characteristic equation

$$
\lambda^{2}+\alpha \lambda+\beta e^{-\lambda r}=0
$$

of the corresponding linearized equation

$$
\ddot{z}(t)+\alpha \dot{z}(t)+\beta z(t-r)=0 .
$$

For first-order equations, results along these lines were recently obtained by $\mathrm{Ku}$ lenović, Ladas, and Meimaridou [2].

A unique feature of our results is that Condition (2) does not have to hold globally but only for values of the argument in some interval. Thus our results apply also in cases where the function $f$ is oscillatory as is the case with the sunflower equation where $f(u)=\sin u$. In particular our results provide a necessary and sufficient condition for the oscillation of all solutions of the sunflower equation with initial function and initial derivative in a certain region.

One of the tools in the proof of our results is the following fixed point theorem of Knaster and Tarski. See [4].

Lemma 1 (Knaster-Tarski Fixed Point Theorem). Let $E$ be a partially ordered set with ordering $\leq$. Assume that inf $E \in E$ and that every nonempty subset of $E$ has a supremum (which belongs to $E$ ). Let $S: E \rightarrow E$ be an increasing mapping (that is, $x \leq y$ implies $S x \leq S y)$. Then $S$ has a fixed point in $E$.

As is customary, a solution $y(t)$ of Eq. (1) is called oscillatory if there exists a sequence of points $\left\{t_{n}\right\}$ such that $\lim _{n \rightarrow \infty} t_{n}=\infty$ and $y\left(t_{n}\right)=0$ for $n=1,2, \ldots$ Otherwise $y(t)$ is called nonoscillatory.

2. Main results. The following result provides sufficient conditions for the oscillation of all solutions of Eq. (1) that lie in a certain strip.

THEOREM 1. Consider the DDE (1), where $\alpha, \beta$, and $r$ are positive constants and $f$ is a real-valued continuous function such that (2) and (3) are satisfied. Assume that the characteristic equation (4) of the linearized equation (5) has no negative roots. Then every solution of Eq. (1) whose graph lies eventually in the strip $R^{+} \times[-A, B]$ is oscillatory.

Proof. Assume, for the sake of contradiction, that Eq. (1) has an eventually positive solution $y(t) \in(0, B]$. Then, in view of $(2)$,

$$
\ddot{y}(t)+\alpha \dot{y}(t)<0,
$$

which implies that the functions

$$
u(t) \equiv \dot{y}(t)+\alpha y(t) \quad \text { and } \quad v(t) \equiv \dot{y}(t) e^{\alpha t}
$$


are decreasing. Let

$$
L_{1}=\lim _{t \rightarrow \infty} u(t) \quad \text { and } \quad L_{2}=\lim _{t \rightarrow \infty} v(t) .
$$

Clearly $L_{1} \in R$, for otherwise $\lim _{t \rightarrow \infty} \dot{y}(t)=-\infty$, which would imply that $\lim _{t \rightarrow \infty} y(t)=-\infty$.

Next, we claim that $L_{2}<0$. Otherwise, $L_{2} \geq 0$ and so eventually $\dot{y}(t) \geq 0$. Thus, $l_{0} \equiv \lim _{t \rightarrow \infty} y(t)$ exists and is positive. Also $l_{1} \equiv \lim _{t \rightarrow \infty} \dot{y}(t)=L_{1}-\alpha l_{0}$ exists. Clearly, $l_{1}$ must be nonnegative, for otherwise $l_{0}$ would be $-\infty$. But from Eq. (1) we have

$$
\lim _{t \rightarrow \infty} \ddot{y}(t)=-\alpha l_{1}-\beta f\left(l_{0}\right)<0,
$$

which implies the contradiction that both $l_{1}$ and $l_{0}$ are $-\infty$. Hence our claim that $L_{2}<0$ has been established. This implies that $\dot{y}(t)<0$, and so $\lim _{t \rightarrow \infty} y(t) \equiv$ $l_{0} \geq 0$. Then $\lim _{t \rightarrow \infty} \dot{y}(t) \equiv l_{1}$ exists, and it must be zero. Otherwise $l_{1}<0$ and so $l_{0}=-\infty$. From (6) we now have $\lim _{t \rightarrow \infty} \ddot{y}(t)=-\beta f\left(l_{0}\right)$, and $l_{0}$ must be zero; otherwise $\lim _{t \rightarrow \infty} \ddot{y}(t)<0$, and hence $l_{0}=-\infty$, which is a contradiction. Therefore we have established that

$$
y(t)>0, \quad \dot{y}(t)<0, \quad \text { and } \quad \lim _{t \rightarrow \infty} y(t)=\lim _{t \rightarrow \infty} \dot{y}(t)=0 .
$$

Next, we claim that there exist positive numbers $m$ and $\varepsilon<\frac{1}{2}$ such that

$$
\lambda^{2}+\alpha \lambda+\beta(1-\varepsilon) e^{-\lambda r} \geq \frac{1}{2} m \text { for } \lambda<0 .
$$

Indeed, by hypothesis, Eq. (4) has no negative roots. Set

$$
F(\lambda) \equiv \lambda^{2}+\alpha \lambda+\beta e^{-\lambda r} \text {. }
$$

Then $F(-\infty)=\infty$ and $F(0)=\beta>0$. It follows that

$$
\lambda^{2}+\alpha \lambda+\beta e^{-\lambda r} \geq m,
$$

where $m=\min _{\lambda<0} F(\lambda)$. Observe that

$$
\lim _{\lambda \rightarrow-\infty}\left(\lambda^{2}+\alpha \lambda+\frac{1}{2} \beta e^{-\lambda r}\right)=\infty
$$

and so there exists $\lambda_{0}<0$ such that

$$
\lambda^{2}+\alpha \lambda+\frac{1}{2} \beta e^{-\lambda r} \geq m / 2 \text { for } \lambda \leq \lambda_{0} .
$$

Choose

$$
\varepsilon=\min \left\{\frac{1}{2}, \frac{m e^{\lambda_{0} r}}{2 \beta}\right\} .
$$

Thus, for $\lambda \leq \lambda_{0}$ using (9) we have

$$
\lambda^{2}+\alpha \lambda+\beta(1-\varepsilon) e^{-\lambda r} \geq \lambda^{2}+\alpha \lambda+\frac{1}{2} \beta e^{-\lambda r} \geq m / 2 .
$$

while for $\lambda_{0}<\lambda<0$ we get

$$
\begin{aligned}
\lambda^{2}+\alpha \lambda+\beta(1-\varepsilon) e^{-\lambda r} & \geq \lambda^{2}+\alpha \lambda+\beta e^{-\lambda r}-\frac{1}{2} m e^{\left(\lambda_{0}-\lambda\right) r} \\
& \geq m-m / 2=m / 2,
\end{aligned}
$$

and the proof of $(8)$ is complete. 
Now, integrating Eq. (1) from $t$ to $\infty$ and using (7), we find

$$
\dot{y}(t)+\alpha y(t)=\beta \int_{t}^{\infty} f(y(s-r)) d s .
$$

Choose $t_{1}$ so large that

$$
\frac{f(y(s-r))}{y(s-r)} \geq 1-\varepsilon \text { for } s \geq t_{1},
$$

which is possible, in view of (3) and (7). Then, for $t \geq t_{1}$ Eq. (10) yields the linear inequality

$$
\dot{y}(t)+\alpha y(t) \geq \beta(1-\varepsilon) \int_{t}^{\infty} y(s-r) d s .
$$

Next, we will establish the following claim.

Claim. Let $y(t)$ be an eventually positive solution of (11). Assume that $\alpha \in R$ and $\beta(1-\varepsilon)>0$. Then

$$
\dot{z}(t)+\alpha z(t)=\beta(1-\varepsilon) \int_{t}^{\infty} z(s-r) d s
$$

has a solution $z(t)$ such that, eventually, $0<z(t) \leq y(t)$.

Proof. Setting $u(t)=y(t) e^{\alpha t}$ into (11), we find that $u(t)>0$ and that

$$
\dot{u}(t) \geq \beta(1-\varepsilon) e^{\alpha t} \int_{t}^{\infty} e^{-\alpha(s-r)} u(s-r) d s .
$$

Integrating from $T$ to $t$, we get

$$
u(t) \geq u(T)+\int_{T}^{t}\left[\beta(1-\varepsilon) e^{\alpha s} \int_{s}^{\infty} e^{-\alpha(\xi-r)} u(\xi-r) d \xi\right] d s
$$

for $t \geq T$. We will employ the Knaster-Tarski fixed point theorem. Let $X$ be the set of all real-valued nondecreasing functions $x$ defined on $[T, \infty)$ and such that

$$
x(t)=u(t) \text { for } T \leq t \leq T+r
$$

and

$$
x(t) \leq u(t) \text { for } t>T+r .
$$

If $x_{1}$ and $x_{2}$ belong to $X$, we will say that $x_{1} \leq x_{2}$ if and only if $x_{1}(t) \leq x_{2}(t)$ for $t \geq T$. Clearly, with this ordering, $X$ is a partially ordered set. Define the mapping $T$ on $X$ as follows:

$$
(T x)(t)=\left\{\begin{array}{l}
u(t), \quad T \leq t \leq T+r \\
u(T+r)+\int_{T+r}^{t}\left[\beta(1-\varepsilon) e^{\alpha s} \int_{s}^{\infty} e^{-\alpha(\xi-r)} x(\xi-r) d \xi\right] d s \\
t>T+r
\end{array}\right.
$$

In view of (15) and (13),

$$
(T x)(t) \leq(T u)(t) \leq u(t) \quad \text { for } t \geq T+r,
$$

while, in view of the definition of $T$,

$$
(T x)(t)=u(t) \text { for } T \leq t \leq T+r .
$$


Also $T x$ is a nondecreasing function for $t \geq T$. Thus, $T: X \rightarrow X$. Finally, inf $X \in X$, and every nonempty subset of $X$ has a supremum that belongs to $X$. Hence, all the hypotheses of Lemma 1 are satisfied, and so $T$ has a fixed point $x \in X$. That is,

$$
(T x)(t)=x(t) \text { for } t \geq T \text {. }
$$

Then

$$
x(t)= \begin{cases}u(t), \quad T \leq t \leq T+r, \\ u(T+r)+\int_{T+r}^{t}\left[\beta(1-\varepsilon) e^{\alpha s} \int_{s}^{\infty} e^{-\alpha(\xi-r)} x(\xi-r) d \xi\right] d s, \quad t>T+r .\end{cases}
$$

Clearly, $x(t)>0$ for $t \geq T$, and differentiating for $t \geq T+r$, we see that $x$ satisfies the equation

$$
\dot{x}(t)=\beta(1-\varepsilon) e^{\alpha t} \int_{t}^{\infty} e^{-\alpha(\xi-r)} x(\xi-r) d \xi, \quad t \geq T+r .
$$

Setting $x(t)=z(t) e^{\alpha t}, t \geq T+r$, we see that $z(t)>0$ and that $z$ satisfies (12). Also, $z(t) e^{\alpha t}=x(t) \leq y(t) e^{\alpha t}$, and the proof of the claim is complete. Finally, differentiating both sides of (12), we see that $z(t)$ is a bounded positive solution of

$$
\ddot{z}(t)+\alpha \dot{z}(t)+\beta(1-\varepsilon) z(t-r)=0 .
$$

This contradicts (8) and the proof is complete.

The following result is a partial converse to Theorem 1 .

THEOREM 2. Consider Eq. (1) and assume that (2) holds,

$$
\begin{gathered}
f(u) \leq u \text { for } u \in[0, B], \\
f \text { is nondecreasing for } u \in[0, B],
\end{gathered}
$$

and the characteristic equation (4) of the "majorant" equation (5) has a real root. Then Eq. (1) has a nonoscillatory solution whose graph lies eventually in the strip $R^{+} \times(0, B]$.

Proof. Let $\mu$ be a real root of Eq. (4). Then clearly $\mu<0$, and so there is a $T \geq t_{0}$ such that $e^{\mu T} \leq B$. Thus, Eq. (5) has the nonoscillatory solution

$$
y(t)=e^{\mu t} \in(0, B] \text { for } t \geq T .
$$

Hence, in light of (16) and (17), the differential inequality

$$
\ddot{y}(t)+\alpha \dot{y}(t)+\beta f(y(t-r)) \leq 0, \quad t \geq T,
$$

is also satisfied by $y(t)=e^{\mu t}$ and (7) holds. Integrating (18) from $t$ to $\infty$ and using (7), we get

$$
\dot{y}(t)+\alpha y(t) \geq \beta \int_{t}^{\infty} f(y(s-r)) d s .
$$

Now, a slight modification in the proof of the claim that we established in the proof of Theorem 1 implies that the equation

$$
\dot{z}(t)+\alpha z(t)=\beta \int_{t}^{\infty} f(z(s-r)) d s
$$


has a solution $z(t)$ such that

$$
0<z(t) \leq e^{\mu t}, \quad t \geq T
$$

Clearly, $z(t)$ is a positive solution of Eq. (1) that lies in the strip $R^{+} \times(0, B]$. The proof is complete.

Combining Theorems 1 and 2, we obtain the following necessary and sufficient condition for the oscillation of Eq. (1).

Theorem 3. Assume that Conditions (2), (3), (16), and (17) are satisfied. Then every solution of Eq. (1) whose graph lies eventually in the strip $R^{+} \times[-A, B]$ oscillates if and only if the characteristic equation (4) of the linearized equation (5) has no negative roots.

3. Application. Consider the so-called sunflower equation

$$
\ddot{y}(t)+\frac{a}{r} \dot{y}(t)+\frac{b}{r} \sin y(t-r)=0,
$$

which describes the helical movement of the tip of growing plants, especially of the sunflower. The study of this problem goes back to the early 1800 s. See [1] and [3] for the description of the model, the study of periodic solutions, and further references.

Somolinos [3] showed that under appropriate initial conditions and with the parameters $a, b$, and $r$ in a certain range the solutions of Eq. (20) remain in the strip $R^{+} \times[-A, B]$, where $A=B=\pi-\varepsilon$ and $\varepsilon>0$ is sufficiently small. Applying our Theorem 3 , we therefore conclude that those solutions oscillate if and only if the characteristic equation

$$
\lambda^{2}+\frac{a}{r} \lambda+\frac{b}{r} e^{-\lambda r}=0
$$

has no negative roots.

\section{REFERENCES}

[1] D. Israelsson and A. Johnsson, A theory of circumnutations of Helianthus annus, Physiol. Plant. 20, 957-976 (1967)

[2] M. R. S. Kulenović, G. Ladas, and A. Meimaridou, On oscillation of nonlinear delay differential equations, Quart. Appl. Math. 45, 155-164 (1987)

[3] A. S. Somolinos, Periodic solutions of the sunflower equation: $\ddot{x}+(a / r) \dot{x}+(b / r) \sin x(t-r)=0$, Quart. Appl. Math. 35, 465-477 (1978)

[4] A. Tarski, A lattice theoretical fixed point theorem and its applications, Pacific J. Math. 5, 285-309 (1955) 